Supporting information for

\title{
Highly efficient and selective metal oxy-boride electrocatalysts for oxygen evolution from alkali and saline solutions
}
Suraj Gupta**, Mark Forster ${ }^{\mathrm{b}}$, Asha Yadav ${ }^{\mathrm{c}}$, Alexander J. Cowan ${ }^{\mathrm{b}}$, Nainesh Patel ${ }^{\mathrm{c}}$, Maulik Patel ${ }^{\mathrm{a}}$

${ }^{a}$ School of Engineering, University of Liverpool, Liverpool, L69 3GH, United Kingdom.

${ }^{b}$ Department of Chemistry and Stephenson Institute for Renewable Energy, University of Liverpool, Liverpool, L69 7ZD, United Kingdom.

${ }^{c}$ Department of Physics, University of Mumbai, Vidyanagari, Santacruz (East), Mumbai 400098, India.

Corresponding author:

Dr. Suraj Gupta

Email: Suraj.Gupta@liverpool.ac.uk, suraj422000@ gmail.com 


\begin{tabular}{|l|c|c|c|c|}
\hline \multirow{2}{*}{ Catalyst } & \multicolumn{3}{|c|}{ Atomic concentration (\%) } & Fe/(Co+Fe) (\%) \\
\cline { 2 - 5 } & Co & Fe & O & \\
\hline Co-O-B & 42.08 & -- & 57.92 & 0.00 \\
\hline Co-Fe-O-B-10 & 24.62 & 2.50 & 72.88 & 9.32 \\
\hline Co-Fe-O-B-20 & 19.56 & 4.37 & 76.07 & 18.26 \\
\hline Co-Fe-O-B-30 & 29.16 & 13.36 & 57.48 & 31.42 \\
\hline Co-Fe-O-B-40 & 15.08 & 8.45 & 76.47 & 35.91 \\
\hline Fe-O-B & -- & 34.69 & 65.31 & 100.00 \\
\hline
\end{tabular}

Table S1: Table representing the actual atomic concentrations of each of the boronized samples, determined from SEM-EDAX. 

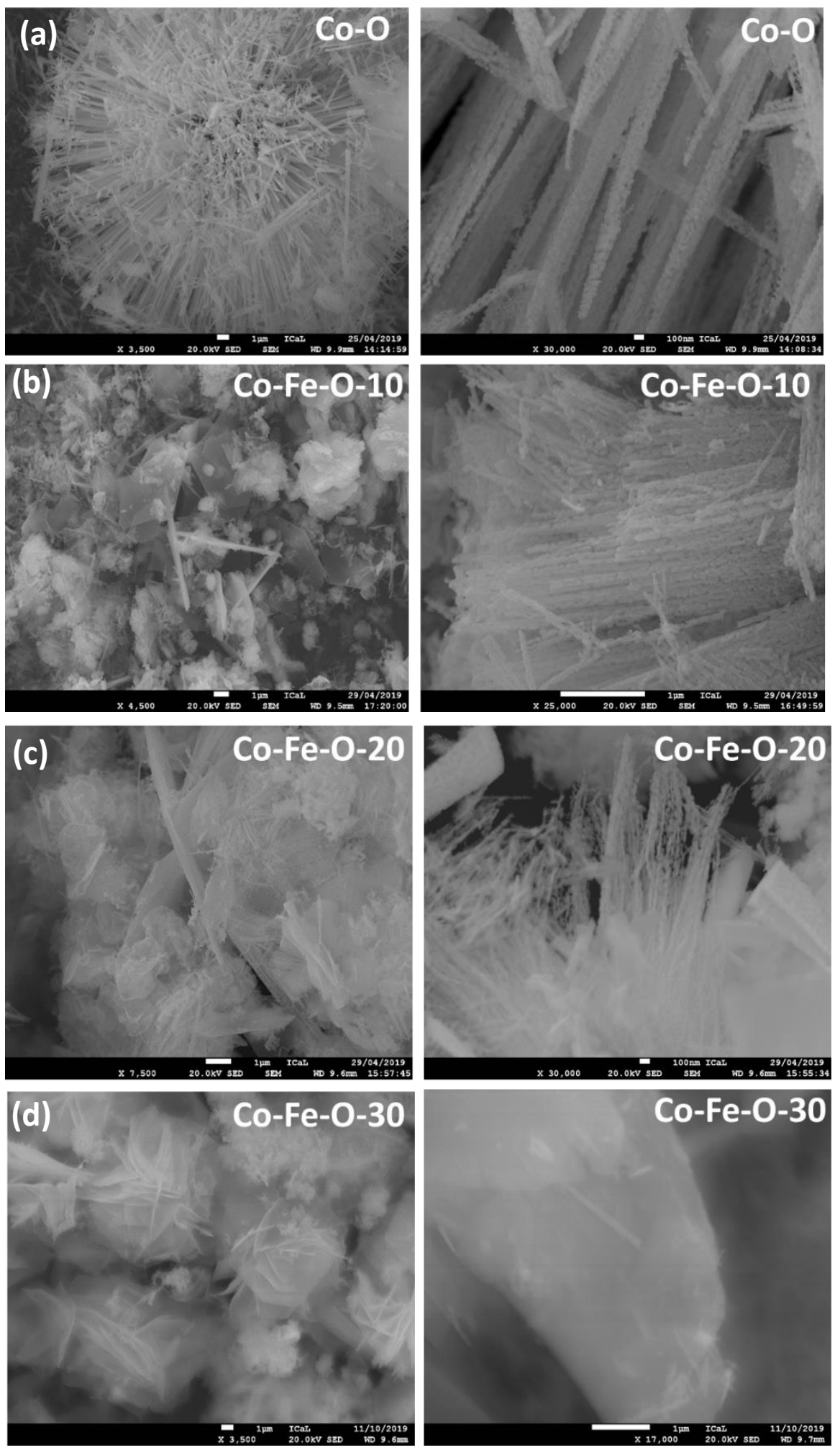

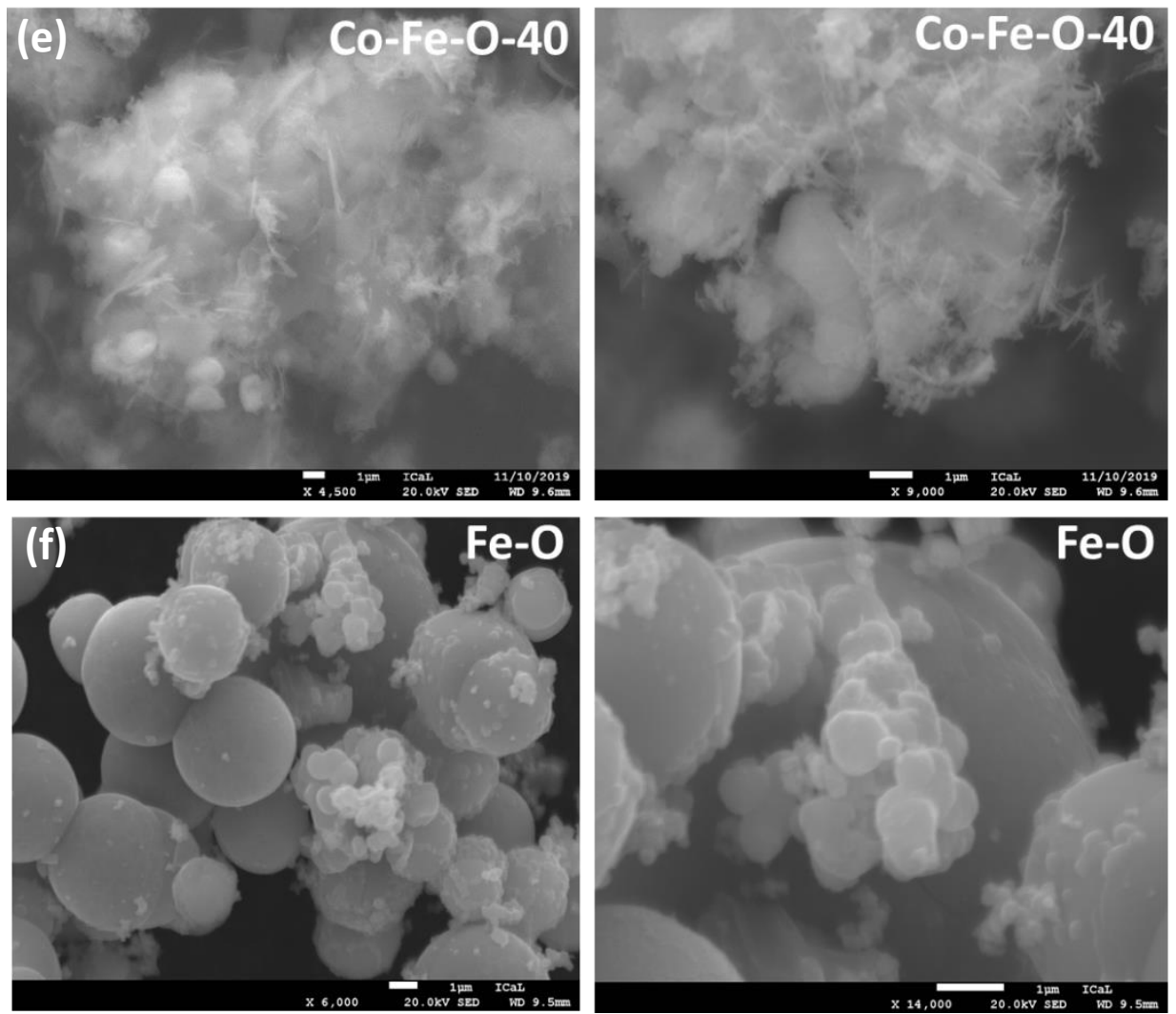

Fig. S1: SEM and HR-SEM images of annealed (a) Co-O; (b) Co-Fe-O-10; (c) Co-Fe-O-20; (d) Co-Fe-O30; (e) Co-Fe-O-40 and (f) Fe-O nanostructures. 


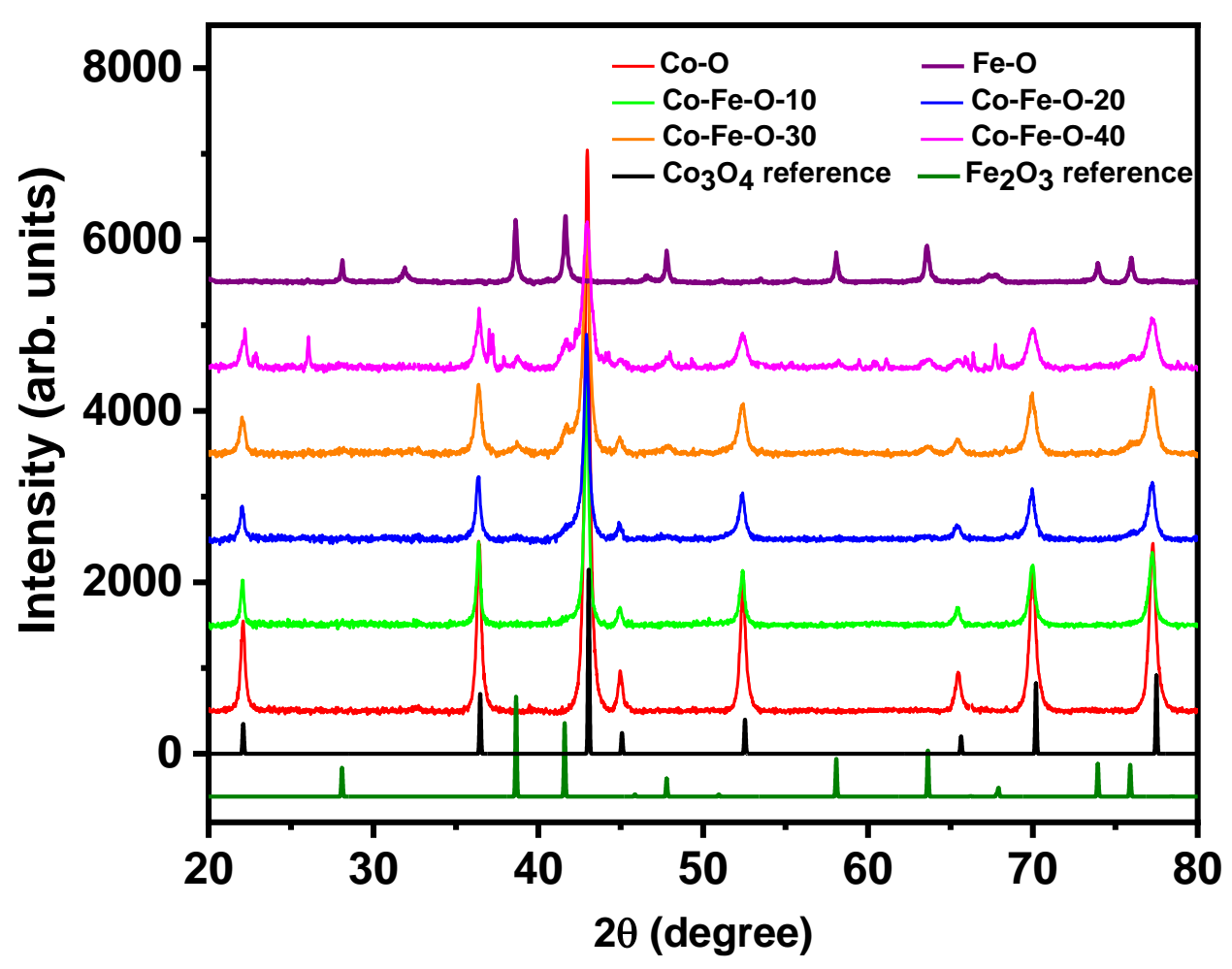

Fig. S2: XRD pattern for Co-O, Co-Fe-O and Fe-O samples along with reference patterns of $\mathrm{CO}_{3} \mathrm{O}_{4}$ and $\alpha-\mathrm{Fe}_{2} \mathrm{O}_{3}$. (The diffraction peaks of $\mathrm{Co}-\mathrm{O}$ at $22.67^{\circ}, 36.45^{\circ}, 43.11^{\circ}, 45.00^{\circ}, 52.55^{\circ}, 65.70^{\circ}, 70.13^{\circ}$ and $77.53^{\circ}$ correspond to reflections from (111), (022), (113), (222), (004), (224), (115) and (044) planes of $\mathrm{CO}_{3} \mathrm{O}_{4}$, respectively). 

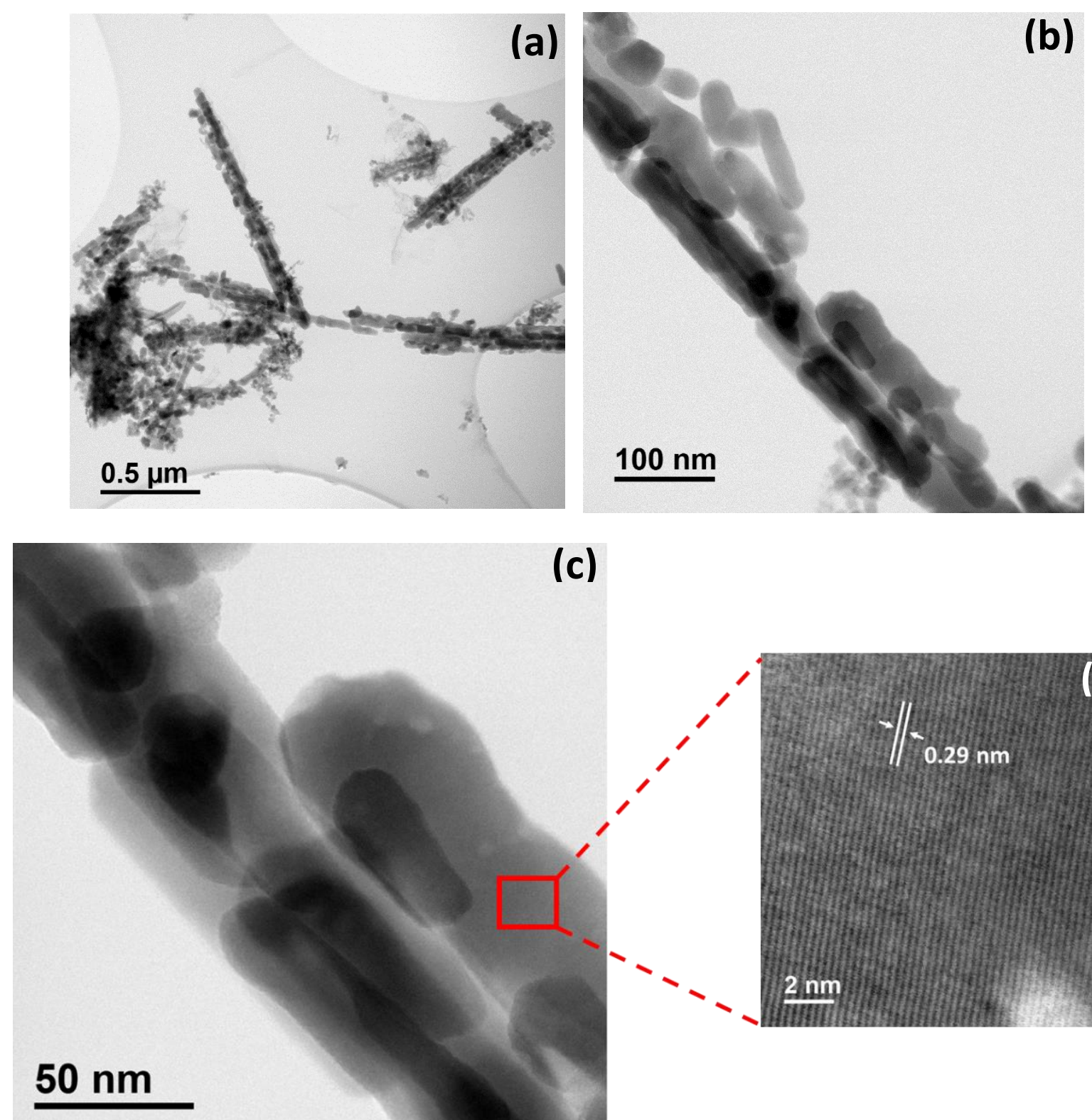

(c)

Fig. S3: (a-c) STEM images of Co-O-B depicting wire-like morphology with nanoparticles as the building block; (d) HR-STEM image showing (022) planes of $\mathrm{CO}_{3} \mathrm{O}_{4}$ on the surface of the wires. 

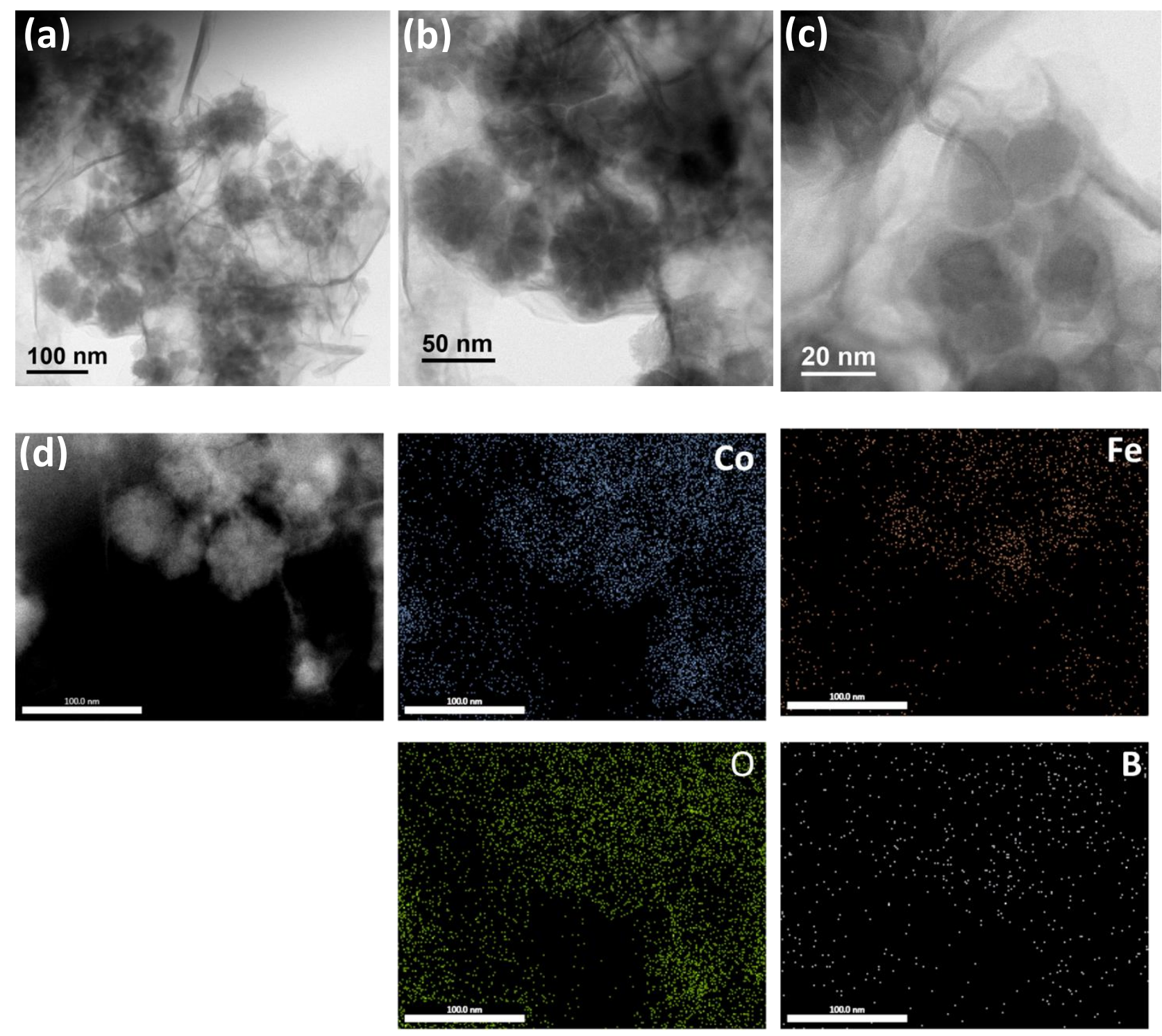

Fig. S4: (a-b) STEM images of Co-Fe-O-B-10 showing flower-like patterns in a thin sheet-like structure; STEM image in (c) shows that the flowers are made up of small core-shell type nanoparticles; (d) STEMEDAX maps of Co-Fe-O-B-10 showing distribution of each of the constituent elements. 

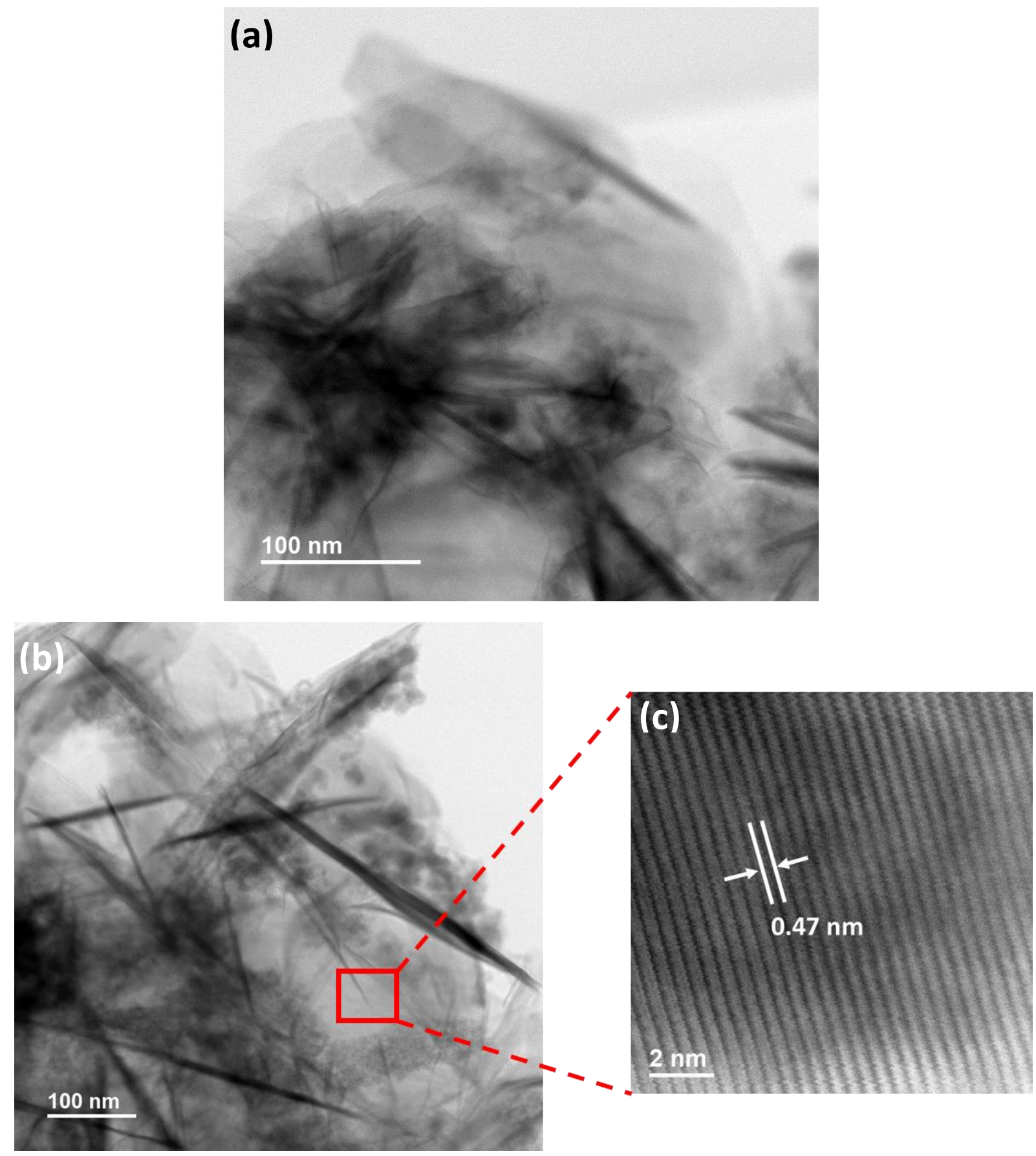

Fig. S5: (a-b) STEM images of Co-Fe-O-B-40 showing graphene-like sheets; (c) HR-STEM image showing (111) planes of $\mathrm{CO}_{3} \mathrm{O}_{4}$ on the surface of the sheets. 

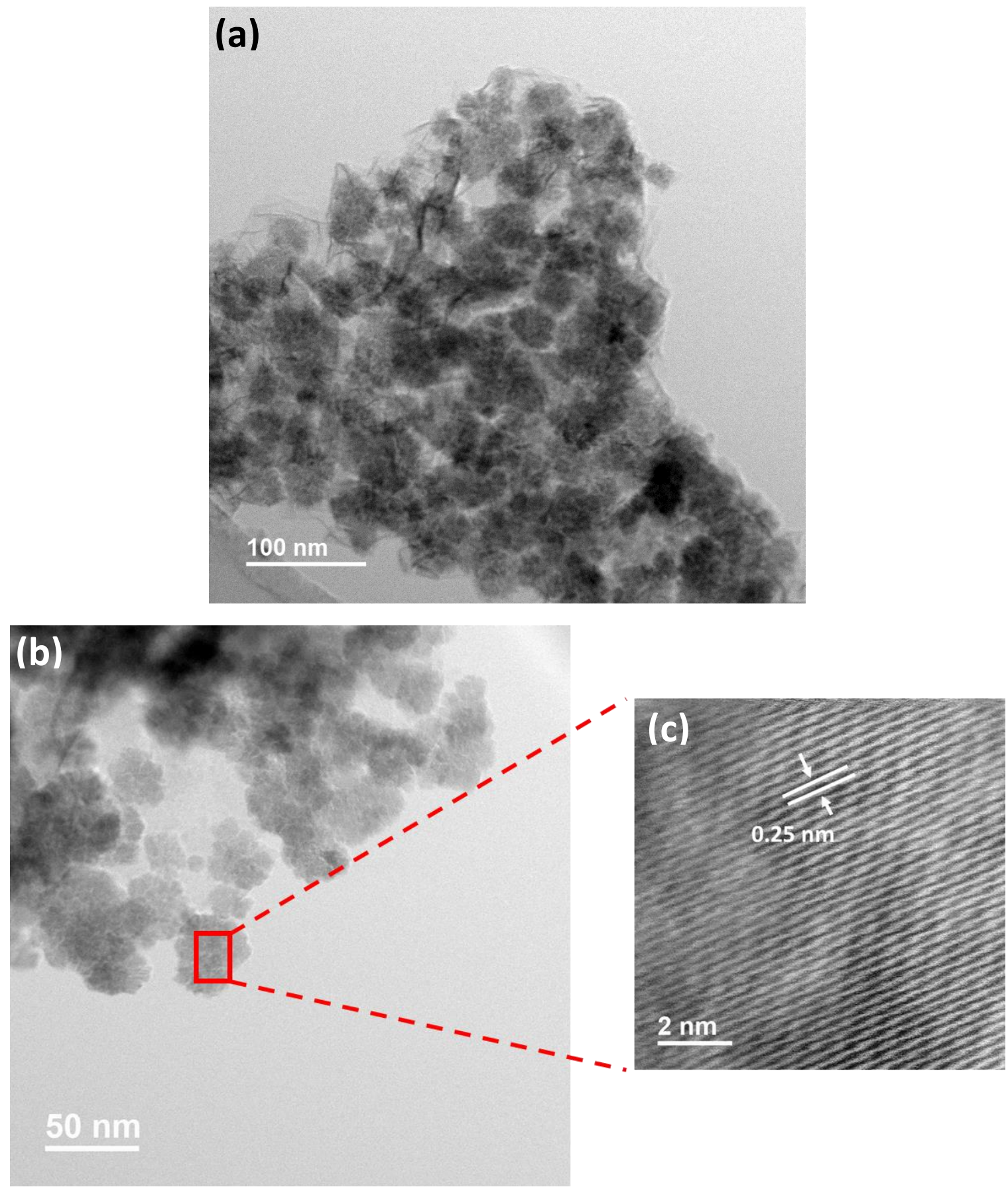

Fig. S6: (a-b) STEM images of Fe-O-B depicting aggregation of small flake-like sheets; (c) HR-STEM image showing (110) planes of $\alpha-\mathrm{Fe}_{2} \mathrm{O}_{3}$ on the surface of the flakes. 

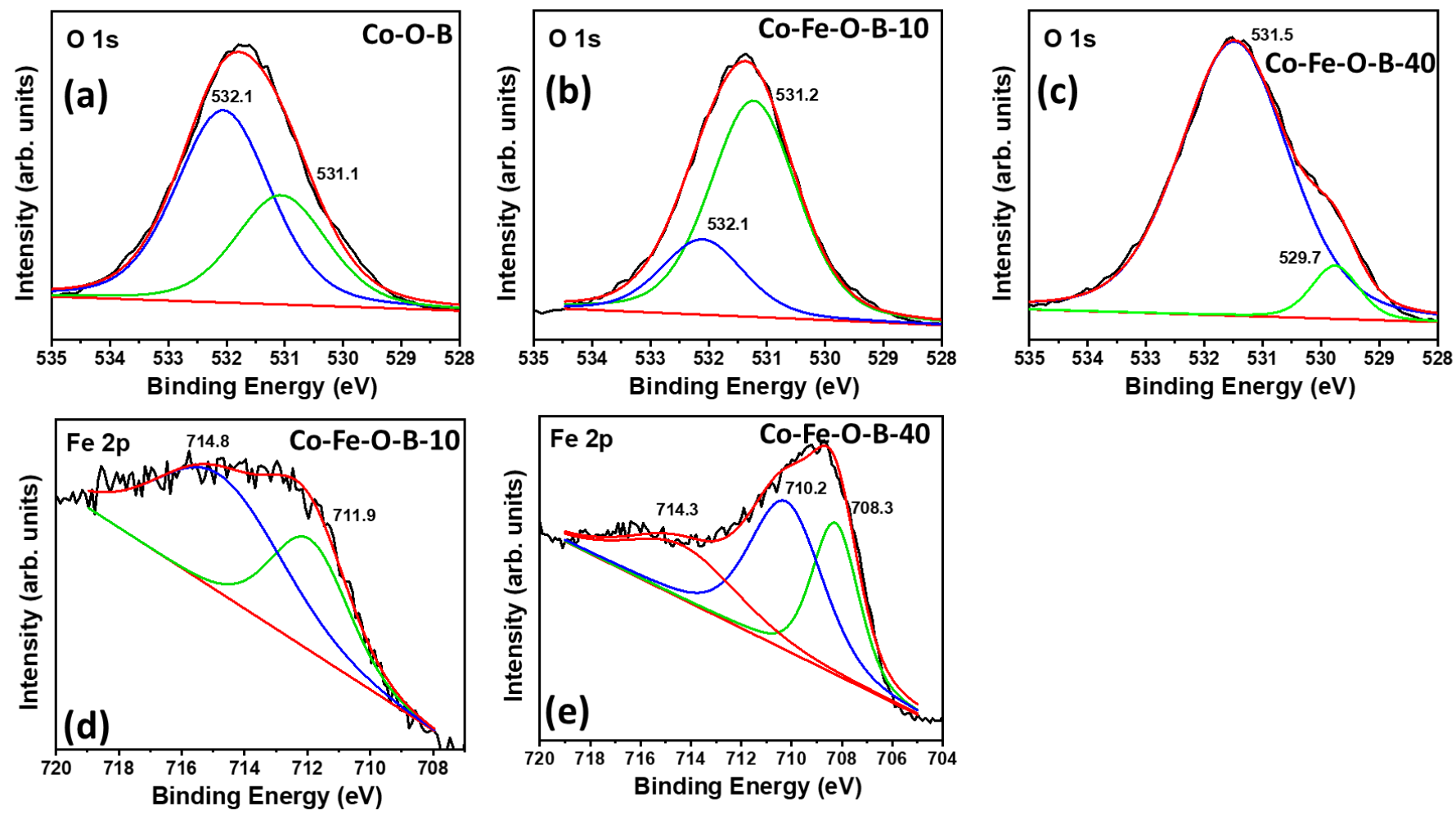

Fig. S7: XPS spectra showing (a-c) O 1s states in Co-O-B, Co-Fe-O-B-10 and Co-Fe-O-B-40 samples; (de) Fe $2 p$ states in Co-Fe-O-B-10 and Co-Fe-O-B-40, respectively. 


\begin{tabular}{|c|c|c|c|c|c|c|}
\hline \multirow[t]{2}{*}{ Samples } & \multicolumn{2}{|c|}{$\begin{array}{l}\text { Over-potential (mV) } \\
\text { at }\end{array}$} & \multicolumn{2}{|c|}{$\begin{array}{l}\text { Tafel Slope } \\
\left(\mathrm{mV} \text { dec }^{-1}\right)\end{array}$} & \multirow{2}{*}{$\begin{array}{l}\text { Double-layer } \\
\text { capacitance } \\
\left(C_{D L}\right)\left(\mathrm{mF} / \mathrm{cm}^{2}\right)\end{array}$} & \multirow[t]{2}{*}{$\begin{array}{l}\text { Rct } \\
(\Omega)\end{array}$} \\
\hline & $\begin{array}{c}10 \mathrm{~mA} \\
\mathrm{~cm}^{-2}\end{array}$ & $\begin{array}{c}100 \mathrm{~mA} \\
\mathrm{~cm}^{-2}\end{array}$ & $\begin{array}{l}\text { Lower } \\
\text { potential }\end{array}$ & $\begin{array}{l}\text { Higher } \\
\text { potential }\end{array}$ & & \\
\hline Co-O-B & 356 & 513 & $53.2 \pm 0.4$ & $278.6 \pm 2.3$ & $20.6 \pm 0.6$ & 38.45 \\
\hline Co-Fe-O-B-10 & 310 & 415 & $47.3 \pm 0.3$ & $237.4 \pm 2.8$ & $12.9 \pm 0.6$ & 16.45 \\
\hline Co-Fe-O-B-20 & 310 & 415 & $50.7 \pm 0.4$ & $209.4 \pm 2.1$ & $18.6 \pm 1.6$ & 20.74 \\
\hline Co-Fe-O-B-30 & 319 & 435 & $49.3 \pm 1.1$ & $211.1 \pm 4.3$ & $20.0 \pm 1.1$ & 31.36 \\
\hline Co-Fe-O-B-40 & 349 & 564 & $93.5 \pm 1.2$ & $532.4 \pm 3.7$ & $4.0 \pm 0.2$ & 87.81 \\
\hline Fe-O-B & 498 & -- & $68.3 \pm 0.7$ & $295.0 \pm 0.7$ & $0.6 \pm 0.03$ & 118.6 \\
\hline $\mathrm{RuO}_{2}$ & 370 & 458 & $90.0 \pm 0.1$ & $79.0 \pm 0.1$ & -- & -- \\
\hline
\end{tabular}

Table S2: Table representing the electrochemical parameters for the boronized samples and $\mathrm{RuO}_{2}$ in $1 \mathrm{M} \mathrm{KOH}$. 


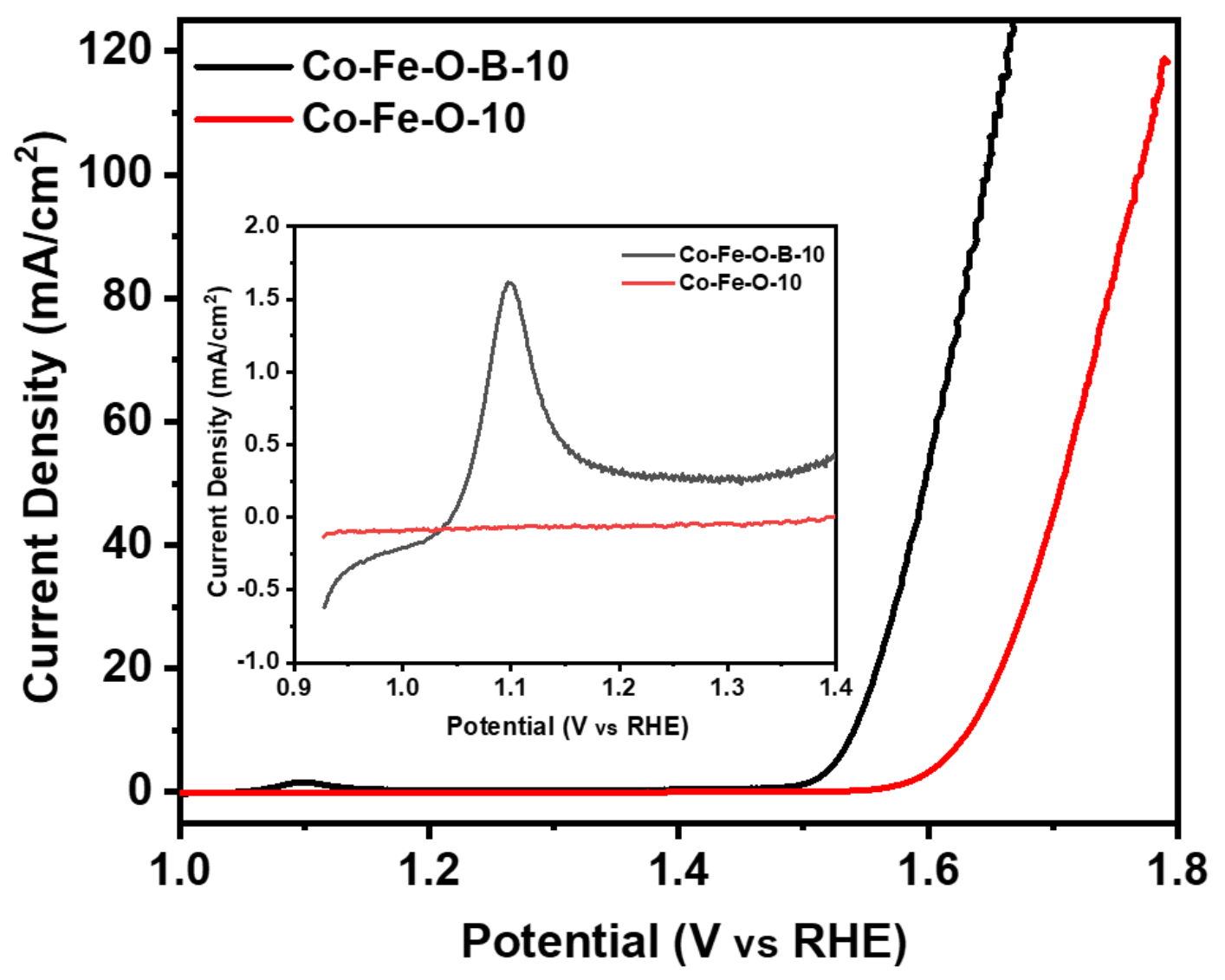

Fig. S8. LSV plots comparing the OER activity of boronized (Co-Fe-O-B-10) and non-boronized (Co-Fe$\mathrm{O}-10$ ) catalysts in $1 \mathrm{M} \mathrm{KOH}$; inset shows the pre-oxidation potential region for the two catalysts. 


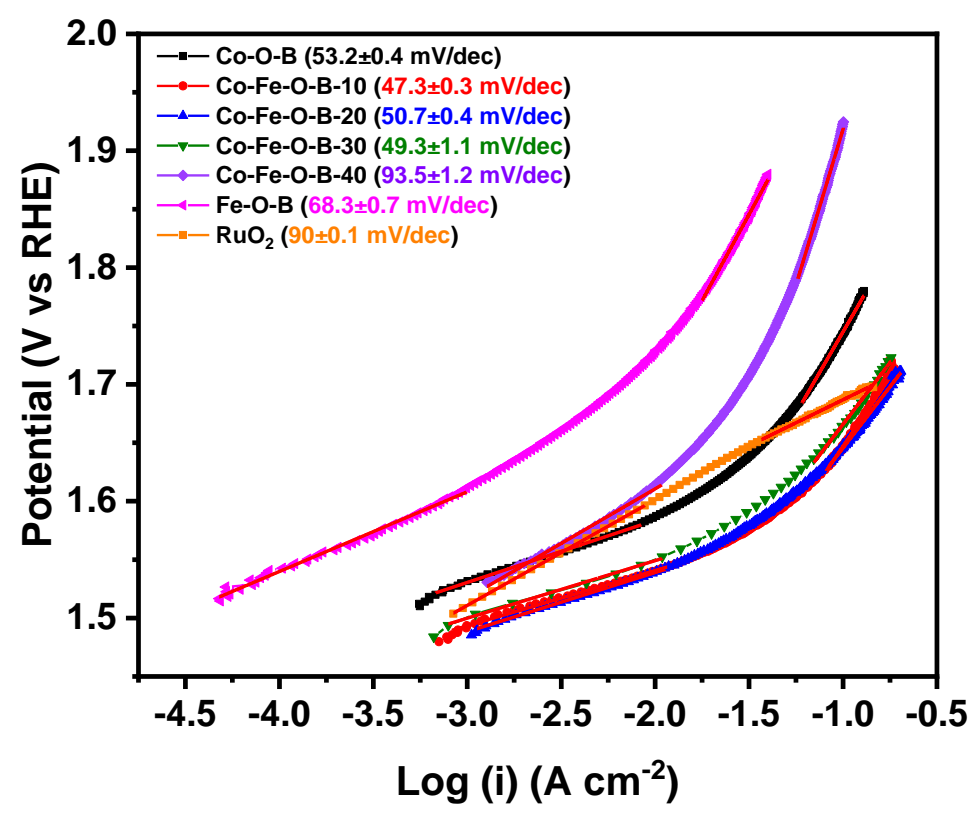

Fig. S9. Tafel plots for Co-O-B, Co-Fe-O-B, Fe-O-B and $\mathrm{RuO}_{2}$ nanocatalysts in $1 \mathrm{M} \mathrm{KOH}$.

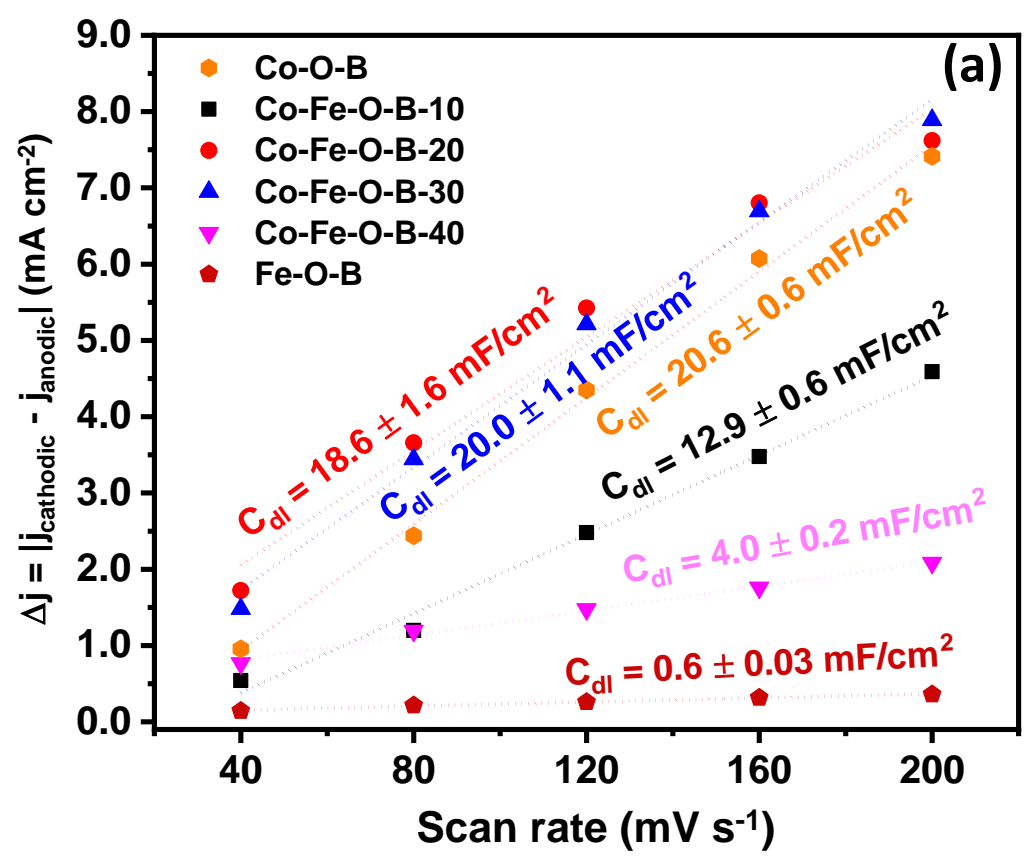



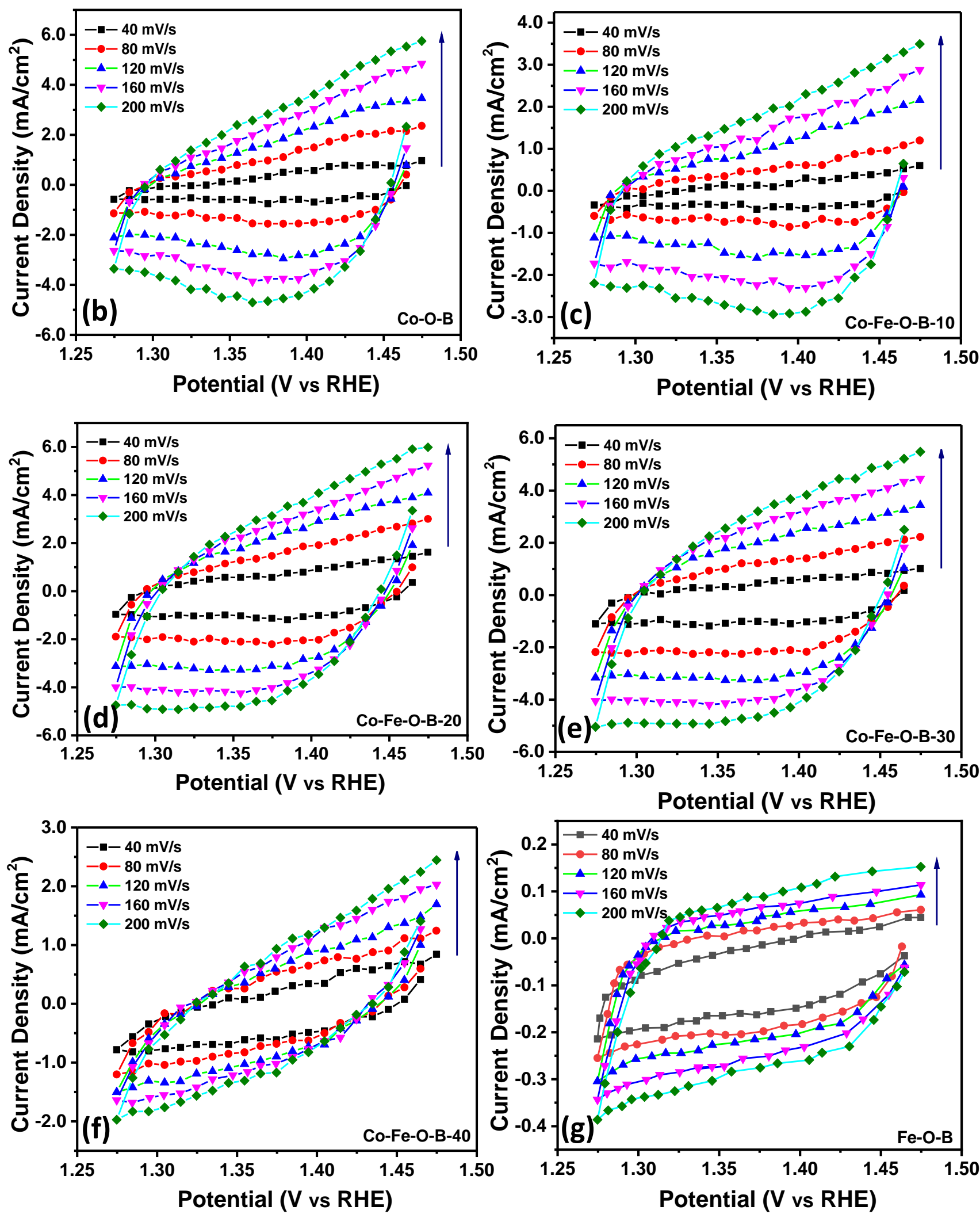

Fig. S10: (a) plot of difference in cathodic and anodic current densities versus the applied scan rates to determine $C_{D L}$ values for Co-O-B, Co-Fe-O-B and Fe-O-B samples; (b-g) CV scans for all samples at varying scan rates from $40 \mathrm{mV} / \mathrm{s}$ to $200 \mathrm{mV} / \mathrm{s}$ in the non-Ohmic potential range to determine $C_{D L}$ values. 


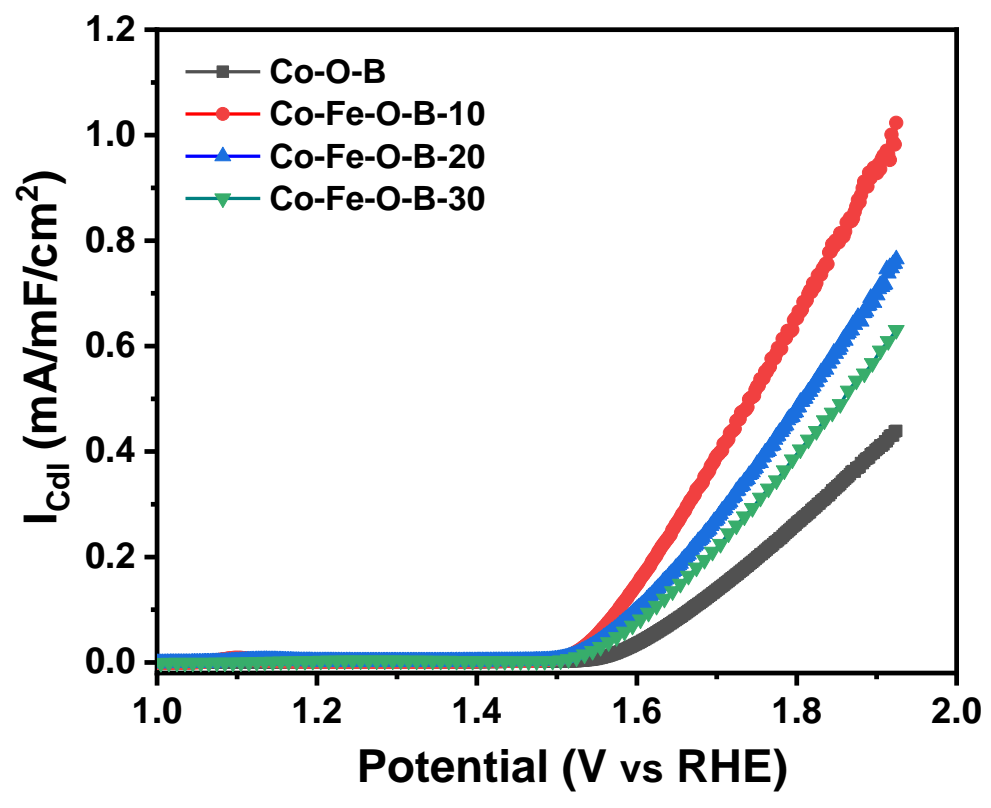

Fig. S11: $C_{D L}$ normalized plots to indicate the intrinsic activity of the Co-rich Co-Fe-O-B samples in alkaline pure water.

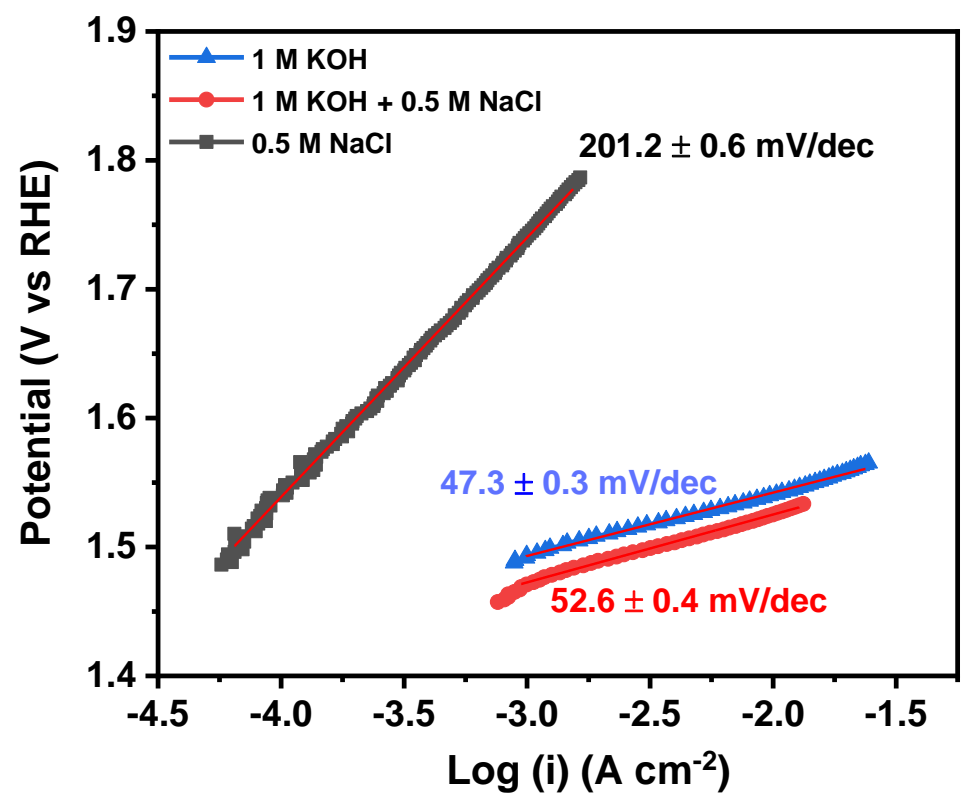

Fig. S12: Tafel plots for Co-Fe-O-B-10 in alkaline pure water, alkaline saline water and neutral saline water electrolytes. 


\begin{tabular}{|c|c|c|c|c|c|c|}
\hline Catalyst & $\begin{array}{l}\text { Substra } \\
\text { te used }\end{array}$ & $\begin{array}{l}\text { Loading } \\
\text { amount } \\
\left(\mathrm{mg} / \mathrm{cm}^{2}\right)\end{array}$ & Electrolyte & $\begin{array}{l}\text { Overpotential } \\
\text { (n) } \\
\text { For OER (mV) }\end{array}$ & $\begin{array}{l}\text { Stability } \\
\text { test }\end{array}$ & $\begin{array}{l}\text { Selectivity } \\
\text { for OER } \\
\text { (FE) }\end{array}$ \\
\hline Co-Fe-O-B* & GC & 0.1 & $\begin{array}{l}1 \mathrm{M} \mathrm{KOH}+ \\
0.5 \mathrm{M} \mathrm{NaCl}\end{array}$ & $\begin{array}{c}\eta_{10}=294 \mathrm{mV} \\
\eta_{100}=434 \mathrm{mV}\end{array}$ & $\begin{array}{c}20 \\
\text { hours }\end{array}$ & $100 \pm 4 \%$ \\
\hline NiFe LDH ${ }^{1}$ & $\mathrm{GC}$ & 0.1 & $\begin{array}{c}0.1 \mathrm{M} \mathrm{KOH}+ \\
0.5 \mathrm{M} \mathrm{NaCl}\end{array}$ & $\eta_{10}=359 \mathrm{mV}$ & 2 hours & $100 \%$ \\
\hline $\begin{array}{c}\text { NiMoN@Ni } \\
\text { FeN }^{2}\end{array}$ & Ni foam & 1270 & $\begin{array}{l}1 \mathrm{M} \mathrm{KOH}+ \\
0.5 \mathrm{M} \mathrm{NaCl}\end{array}$ & $\eta_{100}=286 \mathrm{mV}$ & $\begin{array}{c}100 \\
\text { hours }\end{array}$ & $97.8 \%$ \\
\hline $\begin{array}{c}\mathrm{NiFe} / \mathrm{NiS}_{\mathrm{x}^{-}} \\
\mathrm{Ni}^{3}\end{array}$ & Ni foam & 16 & $\begin{array}{l}1 \mathrm{M} \mathrm{KOH}+ \\
0.5 \mathrm{M} \mathrm{NaCl}\end{array}$ & $\eta_{400}=300 \mathrm{mV}$ & $\begin{array}{l}1000 \\
\text { hours }\end{array}$ & $100 \%$ \\
\hline $\mathrm{NiO}^{4}$ & FTO & -- & $\begin{array}{l}1 \mathrm{M} \mathrm{KOH}+ \\
0.5 \mathrm{M} \mathrm{NaCl}\end{array}$ & $\eta_{10}=340 \mathrm{mV}$ & $\begin{array}{c}100 \\
\text { hours }\end{array}$ & $100 \%$ \\
\hline
\end{tabular}

\section{*This Work}

Table S3: Table comparing the OER performance of recently reported non-noble electrocatalysts in alkaline saline water. 

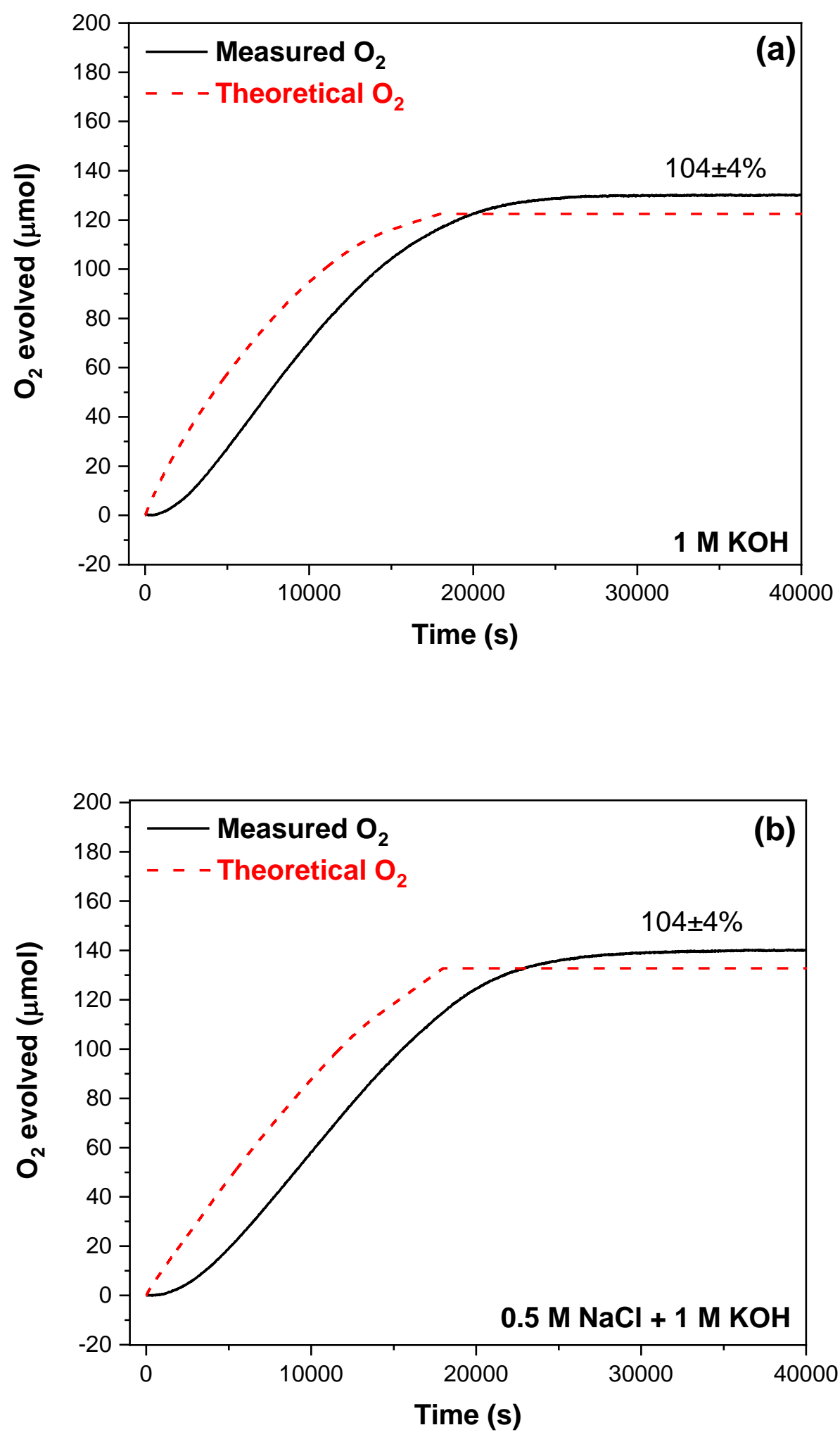

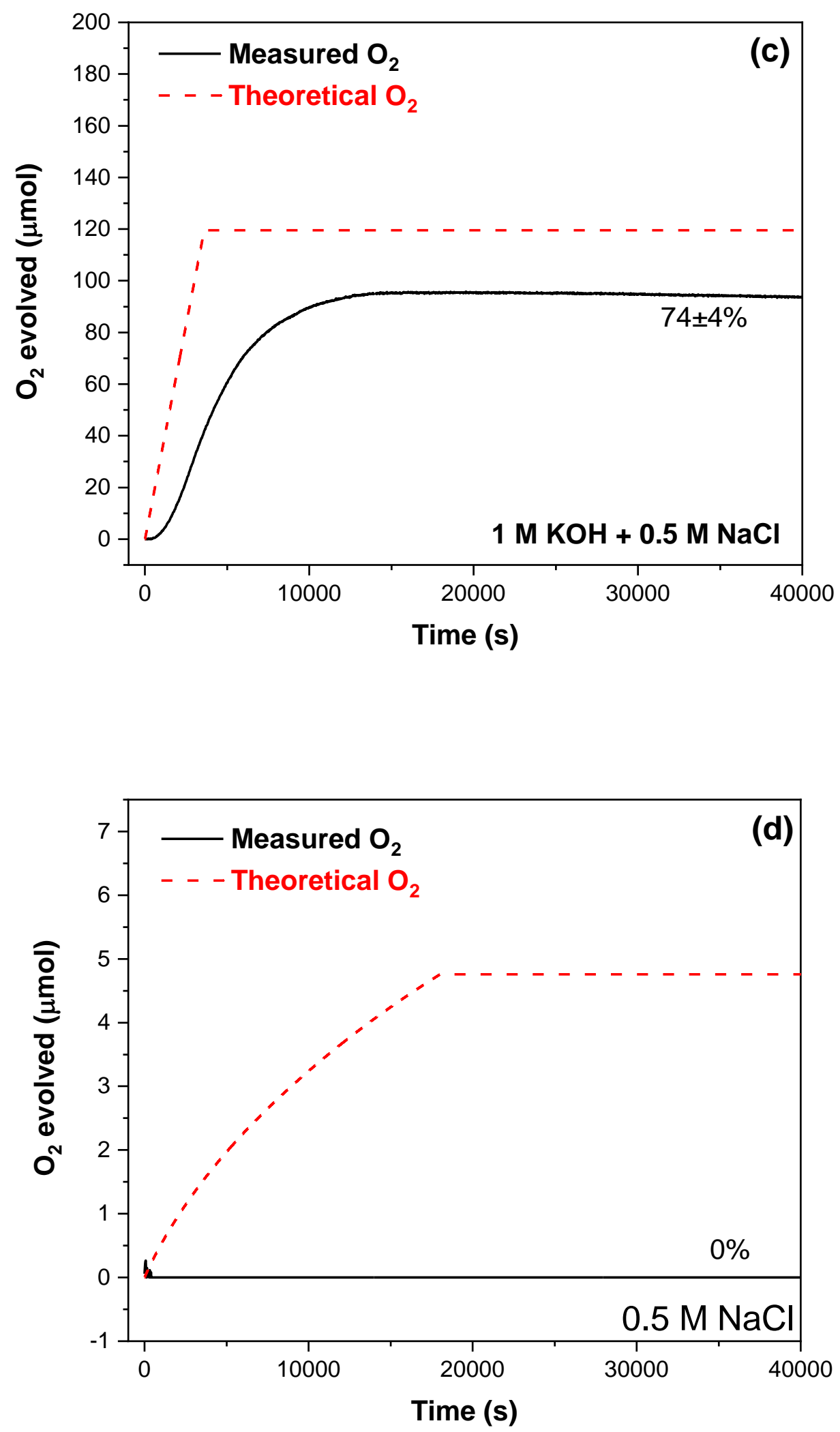


\begin{tabular}{cc}
\hline $\begin{array}{c}\text { Measurement } \\
\text { number }\end{array}$ & Measured $\mathrm{O}_{2} \mathrm{FE} \%$ \\
1 & 100 \\
2 & 103 \\
3 & 97 \\
4 & 106 \\
5 & 109 \\
6 & 104 \\
7 & 104 \\
8 & 104 \\
& \\
Standard & 3.62 \\
deviation $=$ & \\
\hline
\end{tabular}

Fig. S13: Experimental and theoretical values of $\mathrm{O}_{2}$ measured using a fluorescence probe during chronoamperometric tests in (a) alkaline pure water $(1 \mathrm{M} \mathrm{KOH}), 1.65 \mathrm{~V}$ vs RHE for 5 hours; (b) alkaline saline water ( $1 \mathrm{M} \mathrm{KOH}+0.5 \mathrm{M} \mathrm{NaCl}), 1.65 \mathrm{~V}$ vs RHE for 5 hours, (c) alkaline saline water (1 M KOH + $0.5 \mathrm{M} \mathrm{NaCl}$ ) at $100 \mathrm{~mA} \mathrm{~cm}^{-2}$ for 1 hour, (d) neutral saline water (0.5 M NaCl), $2.2 \mathrm{~V}$ vs RHE for 5 hours. As shown in the table, our Faradaic efficiency measurements have a standard deviation of $\sim 4 \%$. 


\begin{tabular}{ccccc}
\hline Electrolyte & & \multicolumn{3}{c}{ Element concentration (mg/L) } \\
\cline { 3 - 5 } & & Co & Fe & B \\
\hline \multirow{2}{*}{$1 \mathrm{M} \mathrm{KOH}$} & Reference & 0.002 & 0.058 & 9.166 \\
\cline { 2 - 5 } & Test & 0.012 & 0.064 & 7.026 \\
\hline $1 \mathrm{M} \mathrm{KOH}+$ & Reference & 0.003 & 0.0063 & 11.768 \\
\cline { 2 - 5 } $0.5 \mathrm{M} \mathrm{NaCl}$ & Test & 0.012 & 0.078 & 8.994 \\
\hline \multirow{2}{*}{$0.5 \mathrm{M} \mathrm{NaCl}$} & Reference & 0.000 & 0.050 & 0.040 \\
\cline { 2 - 5 } & Test & 0.010 & 0.000 & 0.400 \\
\hline
\end{tabular}

\begin{tabular}{|c|c|c|c|c|c|c|c|}
\hline \multicolumn{4}{|c|}{$1 \mathrm{M} \mathrm{KOH} \mathrm{pre-electrochemistry}$} & \multicolumn{4}{|c|}{$1 \mathrm{M} \mathrm{KOH} \mathrm{post-electrochemistry}$} \\
\hline \multicolumn{2}{|c|}{ Element label } & \multirow{2}{*}{$\begin{array}{r}\text { Concentration } \\
9.166(\mathrm{mg} / \mathrm{L})\end{array}$} & \multirow{2}{*}{\begin{tabular}{r|} 
\%RSD \\
0.24
\end{tabular}} & \multicolumn{2}{|c|}{ Element label } & \multirow{2}{*}{$\begin{array}{r}\text { Concentration } \\
7.026(\mathrm{mg} / \mathrm{L})\end{array}$} & \multirow{2}{*}{$\frac{\% \mathrm{RSD}}{0.3}$} \\
\hline B & $(208.889 \mathrm{~nm})$ & & & B & $(208.889 \mathrm{~nm})$ & & \\
\hline B & $(208.956 \mathrm{~nm})$ & $9.186(\mathrm{mg} / \mathrm{L})$ & 0.37 & B & $(208.956 \mathrm{~nm})$ & $7.034(\mathrm{mg} / \mathrm{L})$ & 0.34 \\
\hline B & $(249.678 \mathrm{~nm})$ & $10.41(\mathrm{mg} / \mathrm{L})$ & 0.33 & B & $(249.678 \mathrm{~nm})$ & 7.963 (mg/L) & 0.33 \\
\hline B & $(249.772 \mathrm{~nm})$ & $10.38(\mathrm{mg} / \mathrm{L})$ & 0.33 & B & $(249.772 \mathrm{~nm})$ & 7.943 (mg/L) & 0.25 \\
\hline Co & $(231.160 \mathrm{~nm})$ & $0.002(\mathrm{mg} / \mathrm{L})$ & 14.01 & Co & $(231.160 \mathrm{~nm})$ & $0.012(\mathrm{mg} / \mathrm{L})$ & 5.02 \\
\hline Co & $(236.379 \mathrm{~nm})$ & $0.001(\mathrm{mg} / \mathrm{L})$ & 15.39 & Co & $(236.379 \mathrm{~nm})$ & 0.012 (mg/L) & 4.3 \\
\hline Co & $(237.863 \mathrm{~nm})$ & $0.001(\mathrm{mg} / \mathrm{L})$ & 62.38 & Co & $(237.863 \mathrm{~nm})$ & $0.011(\mathrm{mg} / \mathrm{L})$ & 10.15 \\
\hline Co & $(238.345 \mathrm{~nm})$ & $0.001(\mathrm{mg} / \mathrm{L})$ & 100 & Co & $(238.345 \mathrm{~nm})$ & $0.012(\mathrm{mg} / \mathrm{L})$ & 5.25 \\
\hline $\mathrm{Fe}$ & $(238.204 \mathrm{~nm})$ & $0.058(\mathrm{mg} / \mathrm{L})$ & 1.78 & $\mathrm{Fe}$ & $(238.204 \mathrm{~nm})$ & $0.064(\mathrm{mg} / \mathrm{L})$ & 1.08 \\
\hline $\mathrm{Fe}$ & $(239.563 \mathrm{~nm})$ & $0.059(\mathrm{mg} / \mathrm{L})$ & 2.81 & $\mathrm{Fe}$ & $(239.563 \mathrm{~nm})$ & $0.064(\mathrm{mg} / \mathrm{L})$ & 1.2 \\
\hline $\mathrm{Fe}$ & $(240.489 \mathrm{~nm})$ & $0.058(\mathrm{mg} / \mathrm{L})$ & 2.24 & $\mathrm{Fe}$ & $(240.489 \mathrm{~nm})$ & $0.063(\mathrm{mg} / \mathrm{L})$ & 1.84 \\
\hline
\end{tabular}

\begin{tabular}{|c|c|c|c|c|c|c|c|}
\hline \multicolumn{3}{|c|}{$1 \mathrm{M} \mathrm{KOH}+0.5 \mathrm{M} \mathrm{NaCl}$ pre-electrochemistry } & \multirow{3}{*}{$\begin{array}{r}\text { \%RSD } \\
0.54\end{array}$} & \multicolumn{4}{|c|}{$1 \mathrm{M} \mathrm{KOH}+0.5 \mathrm{M} \mathrm{NaCl}$ post-electrochemistry } \\
\hline \multicolumn{2}{|c|}{ Element label } & \multirow{2}{*}{$\begin{array}{l}\text { Concentration } \\
11.768(\mathrm{mg} / \mathrm{L})\end{array}$} & & & ent label & Concentration & $\%$ RSD \\
\hline $\mathrm{B}$ & $(208.889 \mathrm{~nm})$ & & & $B$ & $(208.889 \mathrm{~nm})$ & $8.994(\mathrm{mg} / \mathrm{L})$ & 0.46 \\
\hline B & $(208.956 \mathrm{~nm})$ & 11.756 (mg/L) & 0.35 & B & $(208.956 \mathrm{~nm})$ & $8.989(\mathrm{mg} / \mathrm{L})$ & 0.4 \\
\hline B & $(249.678 \mathrm{~nm})$ & $13.49(\mathrm{mg} / \mathrm{L})$ & 0.4 & B & $(249.678 \mathrm{~nm})$ & $10.286(\mathrm{mg} / \mathrm{L})$ & 0.34 \\
\hline B & $(249.772 \mathrm{~nm})$ & $13.447(\mathrm{mg} / \mathrm{L})$ & 0.42 & B & $(249.772 \mathrm{~nm})$ & $10.253(\mathrm{mg} / \mathrm{L})$ & 0.33 \\
\hline Co & $(231.160 \mathrm{~nm})$ & $0.003(\mathrm{mg} / \mathrm{L})$ & 30.54 & Co & $(231.160 \mathrm{~nm})$ & $0.012(\mathrm{mg} / \mathrm{L})$ & 9.08 \\
\hline Co & $(236.379 \mathrm{~nm})$ & $0.003(\mathrm{mg} / \mathrm{L})$ & 29.26 & Co & $(236.379 \mathrm{~nm})$ & $0.01(\mathrm{mg} / \mathrm{L})$ & 8.26 \\
\hline Co & $(237.863 \mathrm{~nm})$ & $0.001(\mathrm{mg} / \mathrm{L})$ & 68.42 & Co & $(237.863 \mathrm{~nm})$ & $0.009(\mathrm{mg} / \mathrm{L})$ & 7.29 \\
\hline Co & $(238.345 \mathrm{~nm})$ & $0.002(\mathrm{mg} / \mathrm{L})$ & 60.49 & Co & $(238.345 \mathrm{~nm})$ & $0.009(\mathrm{mg} / \mathrm{L})$ & 7.73 \\
\hline $\mathrm{Fe}$ & $(238.204 \mathrm{~nm})$ & $0.063(\mathrm{mg} / \mathrm{L})$ & 2.47 & $\mathrm{Fe}$ & $(238.204 \mathrm{~nm})$ & 0.078 (mg/L) & 0.94 \\
\hline $\mathrm{Fe}$ & $(239.563 \mathrm{~nm})$ & $0.064(\mathrm{mg} / \mathrm{L})$ & 2.05 & $\mathrm{Fe}$ & $(239.563 \mathrm{~nm})$ & $0.079(\mathrm{mg} / \mathrm{L})$ & 1.28 \\
\hline $\mathrm{Fe}$ & $(240.489 \mathrm{~nm})$ & $0.062(\mathrm{mg} / \mathrm{L})$ & 1.14 & $\mathrm{Fe}$ & $(240.489 \mathrm{~nm})$ & $0.077(\mathrm{mg} / \mathrm{L})$ & 1.86 \\
\hline
\end{tabular}

\begin{tabular}{|c|c|c|c|c|c|c|c|}
\hline \multicolumn{3}{|c|}{$0.5 \mathrm{M} \mathrm{NaCl}$ pre-electrochemistry } & \multirow{3}{*}{$\begin{array}{r}\% \text { RSD } \\
0.73\end{array}$} & \multicolumn{3}{|c|}{$0.5 \mathrm{M} \mathrm{NaCl}$ post-electrochemistry } & \multirow{3}{*}{$\begin{array}{r}\text { \%RSD } \\
0.17\end{array}$} \\
\hline \multicolumn{2}{|c|}{ Element label } & \multirow{2}{*}{$\begin{array}{r}\text { Concentration } \\
0.04(\mathrm{mg} / \mathrm{L})\end{array}$} & & \multicolumn{2}{|c|}{ Element label } & \multirow{2}{*}{$\begin{array}{r}\text { Concentration } \\
0.37(\mathrm{mg} / \mathrm{L})\end{array}$} & \\
\hline B & $(208.956 \mathrm{~nm})$ & & & B & $(208.956 \mathrm{~nm})$ & & \\
\hline B & $(249.678 \mathrm{~nm})$ & 0.04 (mg/L) & 1.55 & B & $(249.678 \mathrm{~nm})$ & $0.4(\mathrm{mg} / \mathrm{L})$ & 0.74 \\
\hline B & $(249.772 \mathrm{~nm})$ & 0.04 (mg/L) & 1.01 & B & $(249.772 \mathrm{~nm})$ & $0.4(\mathrm{mg} / \mathrm{L})$ & 0.51 \\
\hline Co & $(231.406 \mathrm{~nm})$ & 0 (mg/L) & 98.64 & Co & $(231.406 \mathrm{~nm})$ & $0.01(\mathrm{mg} / \mathrm{L})$ & 11.14 \\
\hline Co & $(235.341 \mathrm{~nm})$ & 0 (mg/L) & $>100$ & Co & $(235.341 \mathrm{~nm})$ & $0.01(\mathrm{mg} / \mathrm{L})$ & 38.16 \\
\hline Co & $(237.863 \mathrm{~nm})$ & 0 (mg/L) & $>100$ & Co & $(237.863 \mathrm{~nm})$ & $0.01(\mathrm{mg} / \mathrm{L})$ & 13.99 \\
\hline Co & $(238.892 \mathrm{~nm})$ & 0 (mg/L) & $>100$ & Co & $(238.892 \mathrm{~nm})$ & $0.01(\mathrm{mg} / \mathrm{L})$ & 7.37 \\
\hline $\mathrm{Fe}$ & $(238.204 \mathrm{~nm})$ & 0.05 (mg/L) & 0.88 & $\mathrm{Fe}$ & $(238.204 \mathrm{~nm})$ & 0 (mg/L) & 7.31 \\
\hline $\mathrm{Fe}$ & $(240.489 \mathrm{~nm})$ & 0.05 (mg/L) & 0.49 & $\mathrm{Fe}$ & $(240.489 \mathrm{~nm})$ & 0 (mg/L) & 5.1 \\
\hline $\mathrm{Fe}$ & $(258.588 \mathrm{~nm})$ & 0.05 (mg/L) & 1.3 & $\mathrm{Fe}$ & $(258.588 \mathrm{~nm})$ & 0 (mg/L) & 33.9 \\
\hline $\mathrm{Fe}$ & $(259.940 \mathrm{~nm})$ & $0.05(\mathrm{mg} / \mathrm{L})$ & 1.11 & $\mathrm{Fe}$ & $(259.940 \mathrm{~nm})$ & $0(\mathrm{mg} / \mathrm{L})$ & 19.96 \\
\hline
\end{tabular}

Table S4: ICP-OES analysis of different electrolytes post OER analysis. Reference electrolytes are also tested to report inherent impurities in the salts. (Bottom tables: raw data from the measurement). 

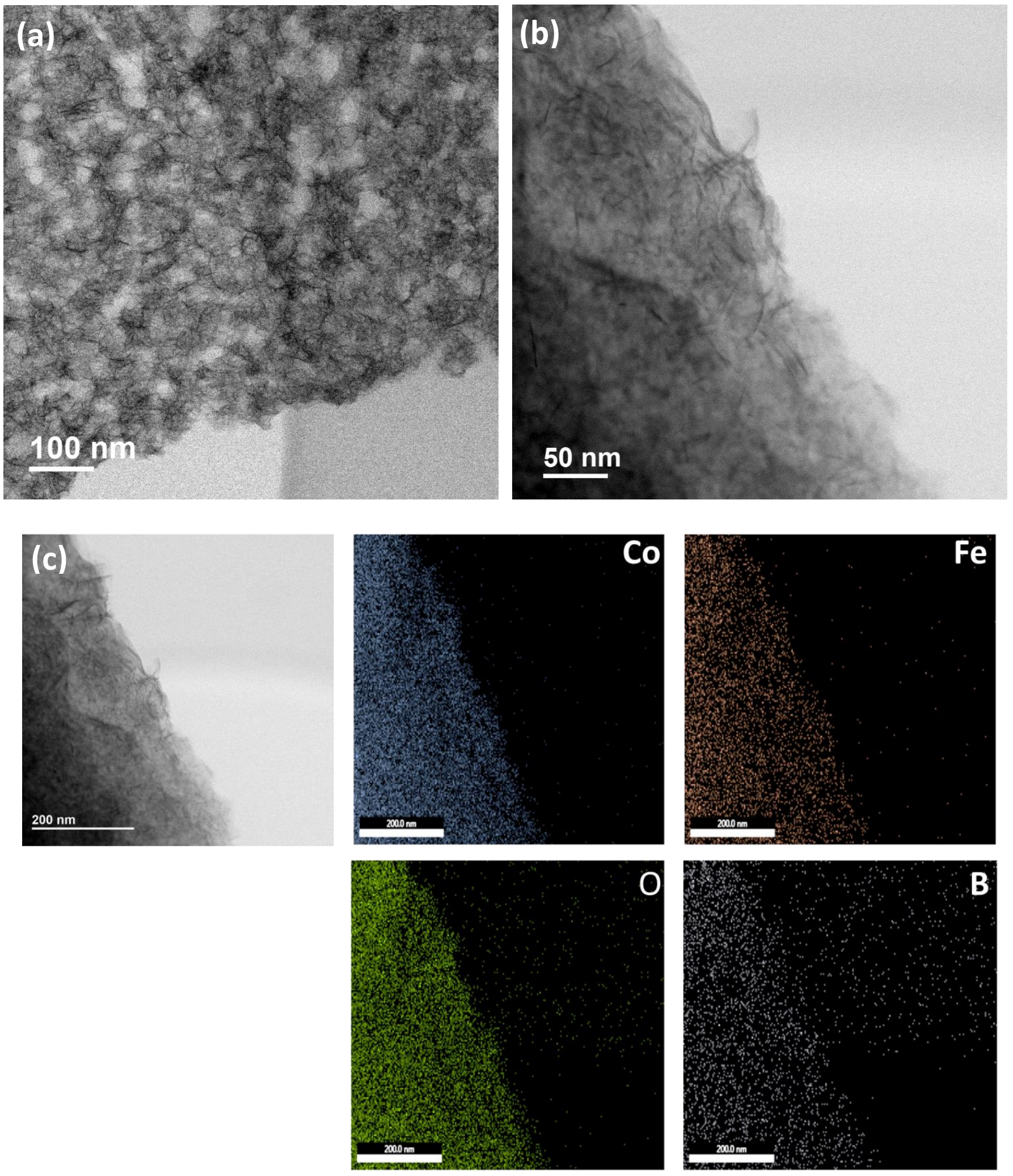

Fig. S14: (a-b) STEM images and (c) STEM-EDAX maps of Co-Fe-O-B-10 after long-term stability test in $1 \mathrm{M} \mathrm{KOH}$ depicting formation of dense cotton-like sheets with uniform distribution of constituent elements and increased concentration of $\mathrm{O}$. 

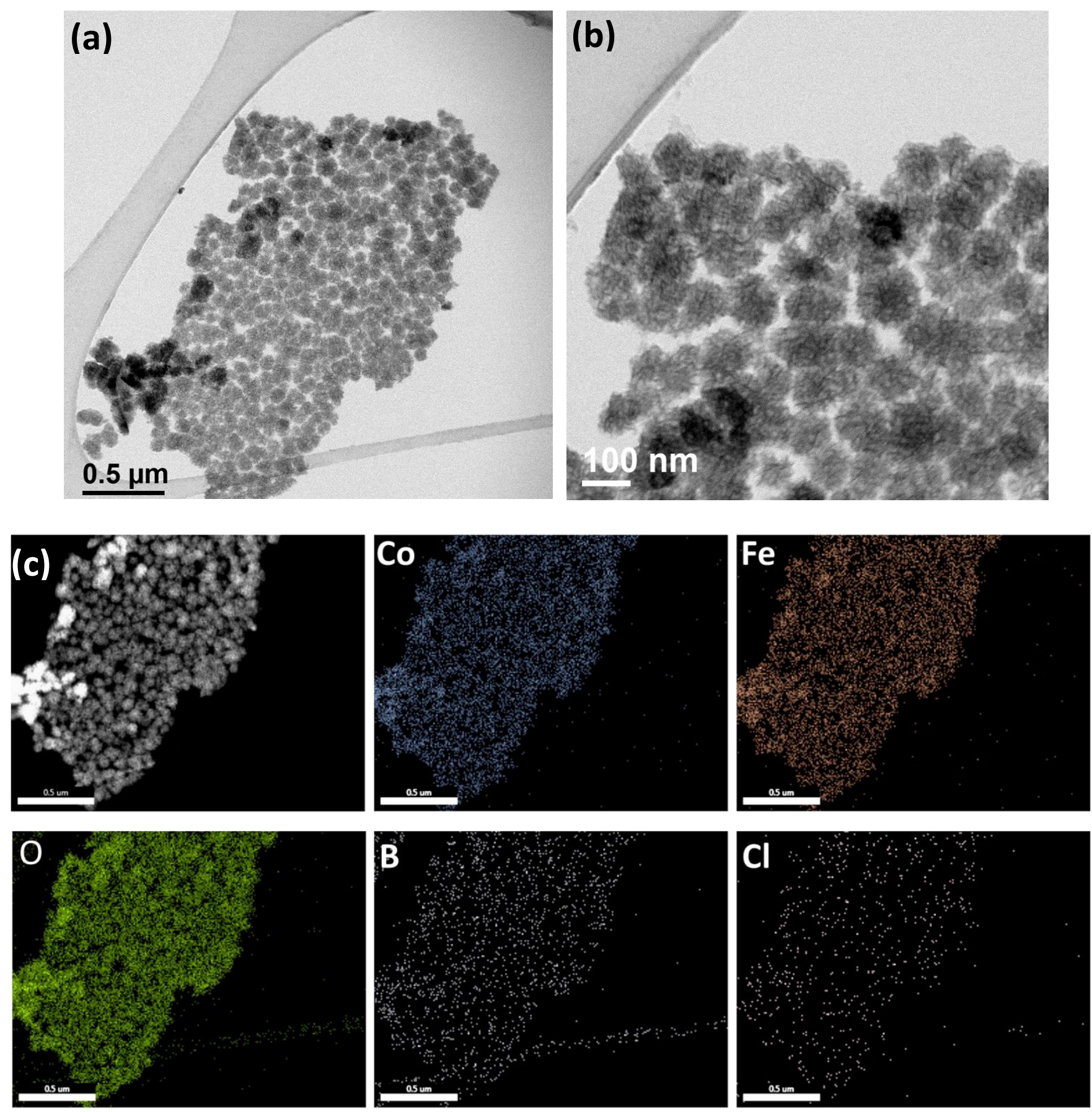

Fig. S15: (a-b) STEM images and (c) STEM-EDAX maps of Co-Fe-O-B-10 after long-term stability test in alkaline saline water $(1 \mathrm{M} \mathrm{KOH}+0.5 \mathrm{M} \mathrm{NaCl})$ depicting that the flower-like morphology is still retained with uniform distribution of constituent elements and increased concentration of $O$. The sample shows $\mathrm{Cl}$ contamination due to the presence of $\mathrm{NaCl}$ in electrolyte. 
(a)

\section{$100 \mathrm{~nm}$}

(b)

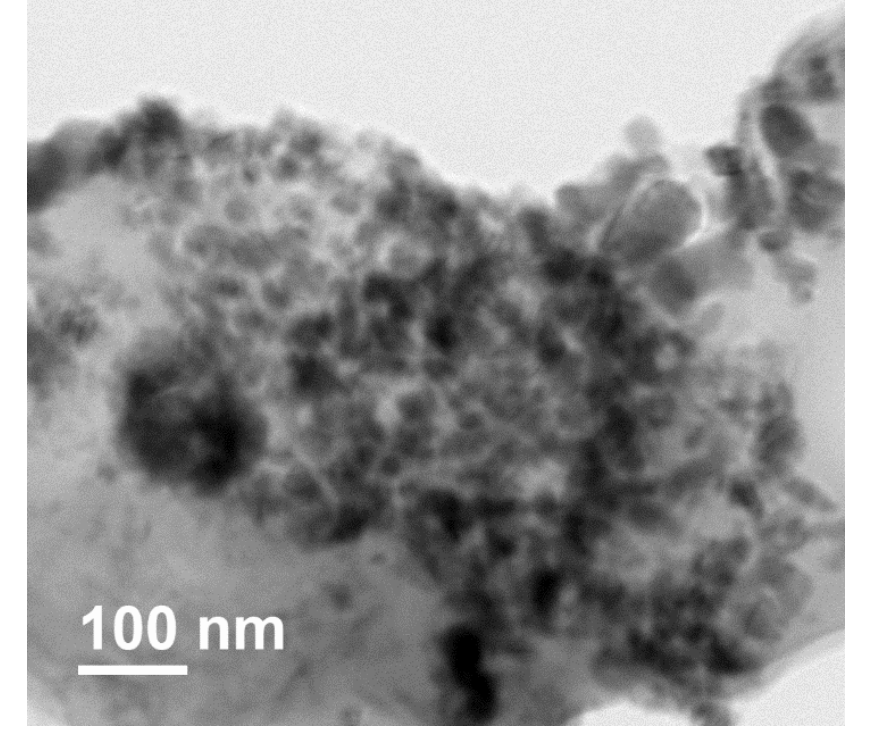

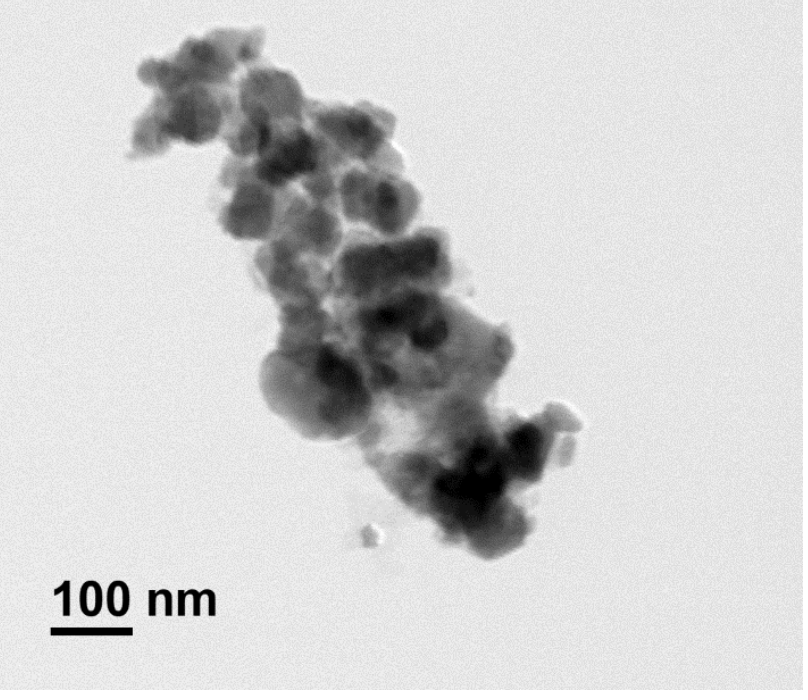
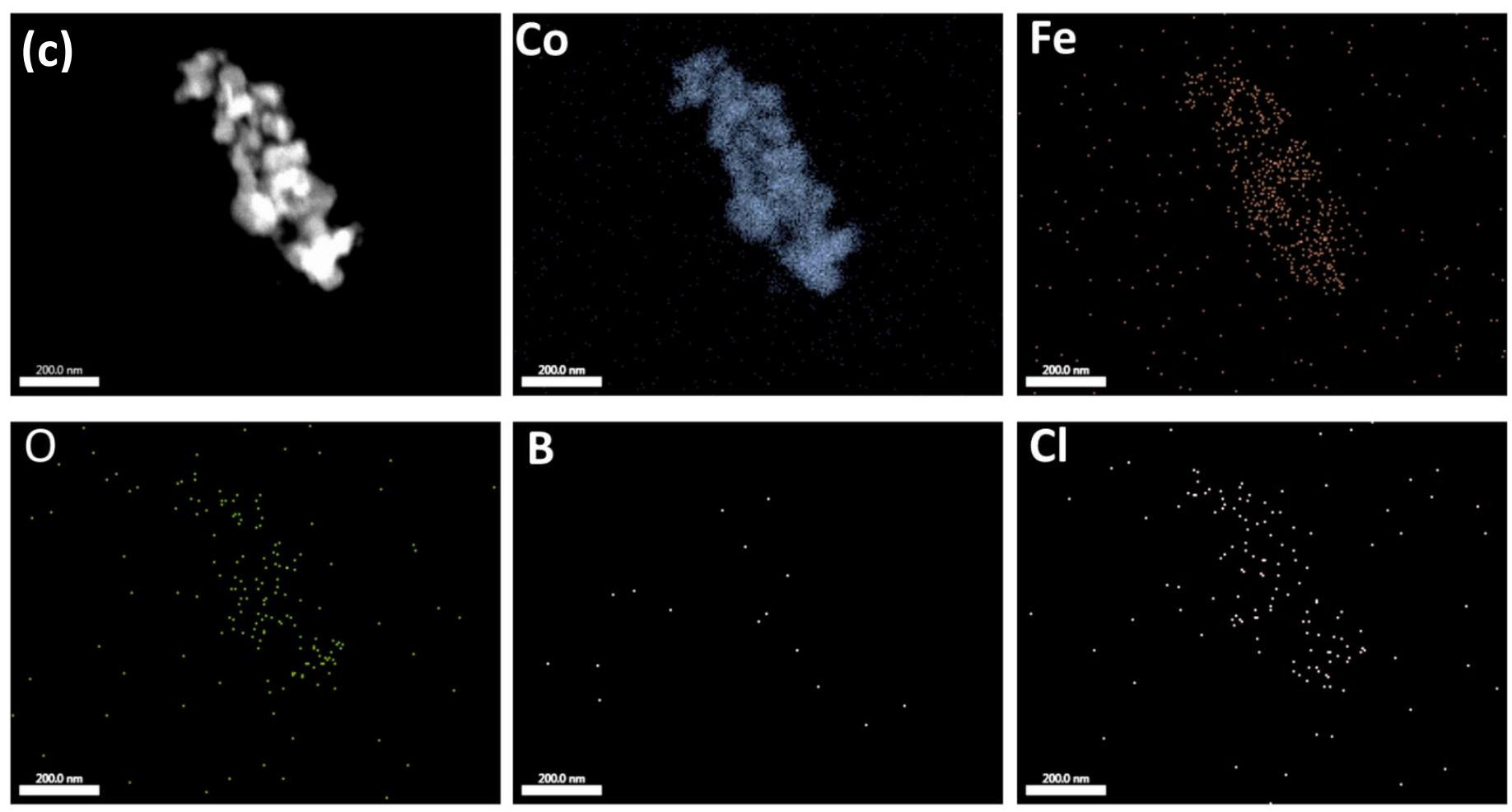

Fig. S16: (a-b) STEM images and (c) STEM-EDAX maps of Co-Fe-O-B-10 after long-term stability test in neutral saline water $(0.5 \mathrm{M} \mathrm{NaCl})$ depicting disarrangement of morphology to form nanoparticles with uniform distribution of $\mathrm{Co}$ and $\mathrm{Fe}$. The samples show very low concentrations of $\mathrm{O}$ and $\mathrm{B}$, while $\mathrm{Cl}$ contamination from electrolyte is also visible. 


\section{References:}

(1) Dionigi, F.; Reier, T.; Pawolek, Z.; Gliech, M.; Strasser, P. Design Criteria, Operating Conditions, and Nickel-Iron Hydroxide Catalyst Materials for Selective Seawater Electrolysis. ChemSusChem 2016, 9 (9), 962-972.

(2) Yu, L.; Zhu, Q.; Song, S.; McElhenny, B.; Wang, D.; Wu, C.; Qin, Z.; Bao, J.; Yu, Y.; Chen, S. NonNoble Metal-Nitride Based Electrocatalysts for High-Performance Alkaline Seawater Electrolysis. Nat. Commun. 2019, 10 (1), 1-10.

(3) Kuang, Y.; Kenney, M. J.; Meng, Y.; Hung, W.-H.; Liu, Y.; Huang, J. E.; Prasanna, R.; Li, P.; Li, Y.; Wang, L. Solar-Driven, Highly Sustained Splitting of Seawater into Hydrogen and Oxygen Fuels. Proc. Natl. Acad. Sci. 2019, 116 (14), 6624-6629.

(4) Juodkazytè, J.; Šebeka, B.; Savickaja, I.; Petrulevičienè, M.; Butkutè, S.; Jasulaitienè, V.; Selskis, A.; Ramanauskas, R. Electrolytic Splitting of Saline Water: Durable Nickel Oxide Anode for Selective Oxygen Evolution. Int. J. Hydrogen Energy 2019, 44 (12), 5929-5939. 